\title{
OPEN Curvature flows, scaling laws and the geometry of attrition under impacts
}

\begin{abstract}
Gergő Pál' ${ }^{1,2}$, Gábor Domokos ${ }^{3,4}$ \& Ferenc Kun ${ }^{1 凶}$
Impact induced attrition processes are, beyond being essential models of industrial ore processing, broadly regarded as the key to decipher the provenance of sedimentary particles. Here we establish the first link between microscopic, particle-based models and the mean field theory for these processes. Based on realistic computer simulations of particle-wall collision sequences we first identify the well-known damage and fragmentation energy phases, then we show that the former is split into the abrasion phase with infinite sample lifetime (analogous to Sternberg's Law) at finite asymptotic mass and the cleavage phase with finite sample lifetime, decreasing as a power law of the impact velocity (analogous to Basquin's Law). This splitting establishes the link between mean field models (curvature-driven partial differential equations) and particle-based models: only in the abrasion phase does shape evolution emerging in the latter reproduce with startling accuracy the spatio-temporal patterns (two geometric phases) predicted by the former.
\end{abstract}

Impact induced damage and fragmentation of solids is ubiquitous in nature and plays a crucial role in the evolution of our geological environment: repeated impacts shape particles (sand grains, pebbles, and volcanic rocks) in sediment transport ${ }^{1-9}$, affect the production of ash and pyroclast particles in volcanic eruptions ${ }^{10,11}$, and contribute to the generation of atmospheric aerosols with consequences on air pollution and on the global climate ${ }^{12}$. In the Solar system, the size and shape of asteroids and of the particles of planetary rings observed today are the results of a long lasting collisional evolution ${ }^{13-17}$. On planet Mars traces of fluvial evolution of landforms such as pebbles have been discovered ${ }^{5,18}$ similar to river beds on Earth. Particle breakage is widely used by the industry in comminution processes of ores and minerals ${ }^{19-23}$, however, it can also be undesired in process and handling engineering due to the resulting degradation of product quality. In these natural processes and industrial applications particles collide both with each other and with hard walls presented by Earth surface (river beds, beaches, and rock walls) or by the components of the process equipment (conveyors, transportation tubes, and containers). A specific area where particle-wall collisions are of utmost importance is the damaging of aircrafts, especially jet engines by impacting hail particles which can cause power reduction and even flame-out of the engine ${ }^{24}$.

Over the past decades, detailed knowledge has been accumulated in geology $y^{1,2,18,25}$, physics ${ }^{26-33}$, and engineering ${ }^{19,23,34-36}$ on single impact breakage phenomena, however, a comprehensive understanding of low velocity impact sequences responsible for the gradual mass reduction and global rounding of solid particles is still lacking. In the physics literature, the existence of two distinct energy phases has been established; these are called the damage phase and the fragmentation phase. These two phases have not only been demonstrated for brittle materials ${ }^{30,32,34,37}$, and plastics spheres ${ }^{38}$, but also for liquid droplets ${ }^{39}$. Moreover, the same two energy phases have also been reported in the geophysics literature ${ }^{8}$ for the collisional attrition of sedimentary particles. In the latter context, global mechanical and geometric understanding of impact induced breakage would be essential to decipher the information hidden in the size and shape of grains and pebbles ${ }^{1-4,18}$. While research in geology and physics concentrated on single impact phenomena, mathematical research related to the proof of the Poincaré conjecture ${ }^{40-42}$ led to the study of a class of nonlinear geometric partial differential equations (PDEs) called curvature-driven flows which appear to be the adequate mean-field theory models for the global evolution of pebbles and other particles under a large number of low energy impacts ${ }^{43,44}$. One may target global shape evolution of particles either by extending the physics literature about single breakage to multiple breakage processes or by relying on mean field PDE models. Although the latter are invaluable tools to obtain qualitative

${ }^{1}$ Department of Theoretical Physics, Doctoral School of Physics, Faculty of Science and Technology, University of Debrecen, P.O. Box 400, 4002 Debrecen, Hungary. ${ }^{2}$ Institute of Nuclear Research (Atomki), P.O. Box 51, 4001 Debrecen, Hungary. ${ }^{3}$ Department of Mechanics, Materials and Structures, Budapest University of Technology and Economics, Müegyetem rkp. 3., K261, 1111 Budapest, Hungary. ${ }^{4}$ MTA-BME Morphodynamics Reserarch Group, Műegyetem rkp. 3., K261, 1111 Budapest, Hungary. ${ }^{\square}$ email: ferenc.kun@science.unideb.hu 
insight, nevertheless, their application has not yet been rigorously justified: until now there existed no theory linking microscopic and macroscopic approaches, in particular, there were no clear physical criteria established for the breakage process which would admit mean field PDEs as valid global approximations.

The latter, on the other hand, appear to be very useful as they make specific geometric predictions: global evolution starting from cuboid polyhedra, serving as averaged models of natural fragments ${ }^{25,45}$, occurs in two geometric phases: in the first, local rounding phase vertices and edges become rounded but axis ratios hardly change while in the second, global rounding phase roundness remains almost constant while axis ratios increase $e^{9,46}$. These geometric phases have been identified both in laboratory experiments and in numerical simulations of the $\mathrm{PDEs}^{9,47}$ and this naturally led to the hypothesis that the geometric phases may also exist in a mechanical abrasion model. In stark contrast to geometric shape evolution of pebbles, no phases can be distinguished in the evolution of mass ${ }^{9}$ which appears to obey Sternberg's empirical law of exponential decay, approaching zero at infinite time ${ }^{48}$.

Here we present a thorough theoretical study of the phase structure of impact induced attrition processes with the primary aim to establish a firm link between microscopic physical breakage models and mean field, macroscopic geometric PDE models. Based on realistic discrete element simulations of sequences of particlewall collisions, we show that, instead of regarding just two distinct energy phases, the damage phase and the fragmentation phase, one has to consider three distinct energy phases since, by regarding impact velocity as a control parameter, the damage phase may be clearly separated into two further energy phases:

- At sufficiently low velocities repeated impacts result in abrasion of the body and lead to a finite asymptotic residual mass; we call this the abrasion energy phase.

- Above a first critical velocity, complete destruction is achieved within a finite number of repetitions; we call this the cleavage energy phase.

The third, highest energy phase, occurring above a second critical velocity corresponds to instantaneous fragmentation where cracks span the entire body and the sample rapidly falls apart into a large number of small pieces; we call this the fragmentation energy phase. The transitions between the abrasion, cleavage, and fragmentation phases occur at two well-defined critical velocities analogous to continuous phase transitions.

We establish the link between microscopic physical breakage models and mean-field PDEs in two steps. First, the splitting of the earlier identified damage phase into the abrasion and cleavage phases delineates the range of validity for the latter: the main feature of the now identified abrasion phase is that each impact removes only a small amount of relative mass. As PDE models are based on the limit where the removed relative mass in each collision approaches zero, our study shows that PDEs can be regarded as a mean field approximation of collision-induced attrition in the abrasion energy phase. Second, we identify one key feature of the PDE model in the microscopic simulation: we show that two geometric phases earlier identified in the context of the PDE model clearly emerge inside the abrasion phase in the microscopic breakage model.

Our finding is based on large scale computer simulations which revealed that the evolution of the mass and shape of the solid is governed by scaling laws in terms of the impact velocity. Most notably, in the abrasion phase the shape evolution of the sample is described by a universal scaling form with a power law dependence on the impact velocity predicting infinite sample lifetime at some finite, asymptotic mass, the latter being determined by the energy threshold for the creation of cracks. In the special limit when this threshold approaches zero, our findings reproduce Sternberg's Law ${ }^{48}$, predicting exponential decay (and infinite lifetime) for sedimentary particles undergoing collisional abrasion in fluvial environments. In addition to verify Sternberg's Law for mass evolution, in the energetic abrasion phase we also confirmed the existence of the two earlier observed geometric phases $^{9,46}$, thus our simulations serve as the first direct mechanical confirmation of curvature-driven PDEs as models of impact-driven abrasion processes. In the cleavage phase we find that the sample lifetime decreases as a power law of the impact velocity analogous to the Basquin law ${ }^{49,50}$ of sub-critical fracture.

\section{Results}

Single impacts: transition from damage to fragmentation. To understand the evolution of solid bodies under repeated collisions with a hard wall, first we focus on single impact events and quantify the resulting mass reduction. We performed numerical measurements by means of computer simulations of a realistic discrete element model (DEM) of body-wall collisions in three dimensions (3D) ${ }^{51-55}$ varying the impact velocity $v_{0}$ in a broad range. To represent freshly fractured rocks with sharp corners and edges in the initial state of shape evolution, rectangular samples of mildly elongated cubic shape were created with the aspect ratio 1:1.2:1.4 of their shortest $c_{0}$, intermediate $b_{0}$, and longest $a_{0}$ sides. This choice is justified by our recent finding that the average shape of fragments is well approximated by a cube for a large diversity of fragmentation processes ${ }^{45}$. The solid was discretized as a random packing of spherical particles, connected by breakable cohesive contacts ${ }^{51-55}$. Parameters of the model were set in such a way that our DEM provides a consistent qualitative and in certain cases quantitative description of the mechanical and fracture properties of the broad class of heterogeneous brittle materials which are abundant in our geological environment ${ }^{30,56-59}$ (see Methods and the Supplementary Information for details of the model construction and parameter settings). Initially, the discretized sample was placed close to a planar wall with a random orientation chosen uniformly on the sphere and the impact was initiated by assigning identical velocity $v_{0}$ perpendicular to the wall to all particles of the solid. As the body moved, it got into contact with the wall and deformed which could result in cracking and fragment formation. The impact lasted until complete rebound was achieved where all particles separated from the wall. In the final state of the process, particles connected by the surviving cohesive elements were identified as fragments. A snapshot of the impact process is presented in Fig. 1. 


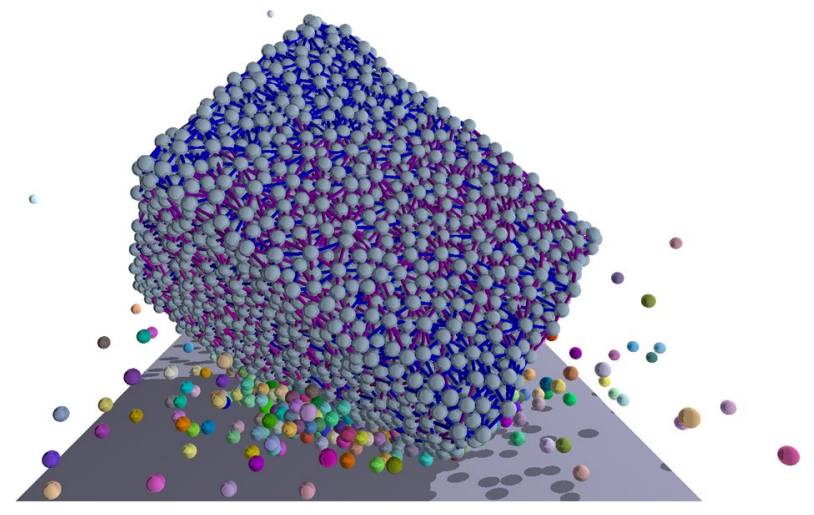

Figure 1. A snapshot of the time evolution of the first impact of a sample with a hard wall. The initially angular body hits the wall close to one of its corners. Most of the fragments are single particles flying at a high speed. Colors are randomly assigned to the fragments. The intact cohesive contacts are represented by lines connecting the spherical particles.

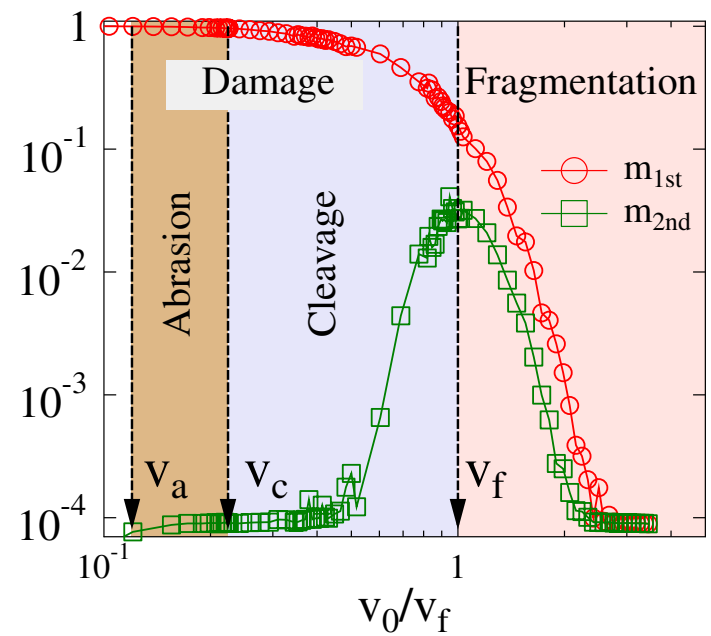

Figure 2. The three energy phases. Mass of the largest $m_{1 s t}$ and second largest $m_{2 n d}$ fragments obtained after a single impact as function of the impact velocity $v_{0}$. In the regime $v_{0}<v_{a}$ of low velocities no cracking occurs and the impactor elastically rebounds from the wall. In the abrasion phase $v_{a}<v_{0}<v_{c}$ small fragments are removed from the body by chipping. To achieve complete breakup in a single impact, $v_{0}$ has to exceed the critical fragmentation velocity $v_{f}$. In the intermediate velocity range $v_{c}<v_{0}<v_{f}$ of cleavage, cracks penetrate deeper inside splitting larger pieces from the body. The critical velocity $v_{c}$ of cleavage is the threshold velocity above which the asymptotic remaining mass tends to zero in repeated collisions. Horizontal axis shows on logarithmic scale the impact velocity $v_{0}$ normalized by critical fragmentation velocity $v_{f}$. For the model solid we found the (non-dimensional) ratio of threshold velocities to be $v_{a} / v_{f}=0.124$. and $v_{c} / v_{f}=0.224$.

Simulations revealed that for sufficiently low impact velocities $v_{0}<v_{a}$ the sample solely underwent deformation around the impact site and rebounded elastically without suffering any damage. Cracks first occurred when $v_{0}$ surpassed a threshold velocity $v_{a}$ determined by the strength of the internal cohesive elements of the material. In this low velocity range, deformation and crack formation is restricted to the vicinity of the contact zone, while for high impact velocities cracks can span the entire sample giving rise to rapid breakup. To give a quantitative characterization of the final outcome and the degree of destruction caused by impacts, we determined the average masses $M_{1 s t}$ and $M_{2 n d}$ of the largest and second largest fragments, respectively. After normalizing these values by the total mass $M_{0}$ we plotted $m_{1 s t}=\left\langle M_{1 s t} / M_{0}\right\rangle, m_{2 n d}=\left\langle M_{2 n d} / M_{0}\right\rangle$ as function of the impact velocity $v_{0}$. It can be observed in Fig. 2 that at low impact velocities we have $m_{2 n d} \ll m_{1 s t}$, i.e. the second largest fragment is orders of magnitude smaller than the largest one, showing that only small pieces are removed from the body around the impact site. This is characteristic for the damage energy phase. Fragmentation is achieved when the second largest piece becomes comparable to the largest one, which first occurs at the maximum of $m_{2 n d}$ defining the critical velocity $v_{f}$ of fragmentation. Beyond the critical fragmentation velocity $v_{f}$ both $m_{1 s t}$ and $m_{2 n d}$ decrease monotonically. Figure 2 shows that, depending on the velocity, single impacts give rise either to damage or fragmentation of the sample with a sharp transition at the critical velocity $v_{f}$. The damage-fragmentation transition 


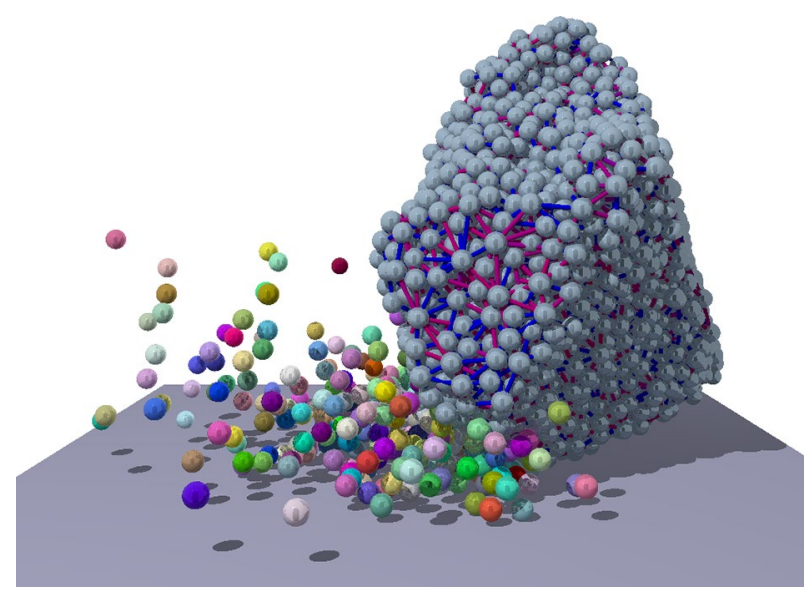

Figure 3. A snapshot of the 8 th impact of a residue at the velocity $v_{0} / v_{f} \approx 0.4$ using the same representation of fragments and cohesive contacts as in Fig. 1. The original edges have been already rounded due to mass removal.

has already been studied in experiments and computer simulations of impacting spherical samples against a hard wall, using heterogeneous brittle materials ${ }^{30,32,34,37}$, plastics spheres ${ }^{38}$, and liquid droplets ${ }^{39}$. In these studies, the same qualitative behavior was obtained for the largest fragment masses $m_{1 s t}, m_{2 n d}$ as in Fig. 2, which implies that the overall outcome of the process in the high velocity range is entirely controlled by the impact velocity and its critical value $v_{f}$, whereas neither the sample's shape nor materials' features have any relevant effect. The detailed analysis of the mass distribution of fragments revealed that the observed universality is caused by the underlying continuous phase transition from damage to fragmentation as the impact velocity is varied ${ }^{26,39,60}$. The identification of the known damage and fragmentation phases also serves as a verification of our model.

Repeated impacts and the two sub-phases of damage: abrasion and cleavage. In the previous subsection, confirming earlier results, we established for single impact phenomena the existence of the two main energy phases. Now we will show that, if we consider not just a single impact but impact sequences, the damage phase can be subdivided into two narrower energy phases: abrasion and cleavage. The damage phase, characterized by $v_{0} \ll v_{f}$ is often observed in natural and industrial processes at lower energy levels. Under such conditions, the large residue of the sample typically undergoes repeated collisions which give rise to a complex evolution of its size and shape. In the following we extend the global phase diagram of Fig. 2 refining the structure of the damage phase by characterizing qualitatively different evolution histories of residues under repeated sub-critical impacts.

To simulate sequences of particle-wall collisions, in the final state of an impact event we identified the largest fragment as the residue of the body, which was further processed to obtain a completely relaxed object for the initial state of the next impact (see "Methods" for the details of the preparation of the residue). The residue was subsequently randomly rotated in three dimensions and was impacted against the wall with the same impact velocity $v_{0}<v_{f}$ as before. The above procedure was repeated up to $N_{\max }=400$ times, or until complete destruction of the body, at $\approx 60$ different impact velocities, respectively. As an example, Fig. 3 illustrates the 8th impact of a residue. For each sequence, 120 different initial samples were used, while in subsequent impacts the residues were randomly rotated by uniformly choosing a direction on the sphere. These calculations revealed an astonishingly rich phase structure of the sub-critical $v_{0}<v_{f}$ regime.

To quantify the gradual mass reduction during the collision sequence, Fig. 4a presents the average $m_{r}=\left\langle M_{r} / M_{0}\right\rangle$ of the residual mass $M_{r}$ normalized by the initial mass of the sample $M_{0}$, as a function of the impact number $N$ for several values of $v_{0}$. We remark that for a single impact event we have $m_{r} \equiv m_{1 s t}$. At very low velocities $v_{0} \ll v_{f}$, a single impact always gives rise only to a few fragments which are typically single spheres, i.e. powder in the model. As a consequence, in Fig. 4 a the residual mass $m_{r}$ gradually decreases with increasing impact number $N$, however, mass reduction gets limited for high $N$ values and a finite asymptotic residual mass emerges $m_{r} \rightarrow m_{r}^{a}$ as $N \rightarrow \infty$. The reason is that due to the decreasing mass $M_{r}$, the kinetic energy $E_{0}=\frac{1}{2} M_{r} v_{0}^{2}$, imparted to the sample decreases, since the impact velocity $v_{0}$ is fixed. Consequently, beyond a certain impact number, i.e. below a certain value of $M_{r}$, the emerging deformation is not sufficient to induce further cracking.

Since only small pieces are removed in single impacts, we term this velocity regime as the abrasion phase of the system characterized by the existence of a finite asymptotic residual mass $m_{r}^{a}>0$. It can be observed in Fig. 4a that the value of $m_{r}^{a}$ decreases with increasing impact velocity $v_{0}$. The value of $m_{r}^{a}$ depends also on the energy threshold for the creation of cracks, i.e. on the strength of cohesive contacts. In the limit when this threshold approaches zero, our findings reproduce Sternberg's Law ${ }^{48}$, predicting exponential decay to zero mass and infinite lifetime for sedimentary particles undergoing collisional abrasion in fluvial environments.

When $v_{0}$ gets sufficiently high, the functional form of $m_{r}(N)$ qualitatively changes: the mass of the residue sets to a rapid decrease with $N$, and repeated impacts give rise to a complete destruction of the sample within a finite number of repetitions. This behavior is characterized by impact velocities in the range $v_{c}<v_{0}<v_{f}$ and we call this interval the cleavage phase of the impact sequence. The critical velocity $v_{c}$ of cleavage is defined as 

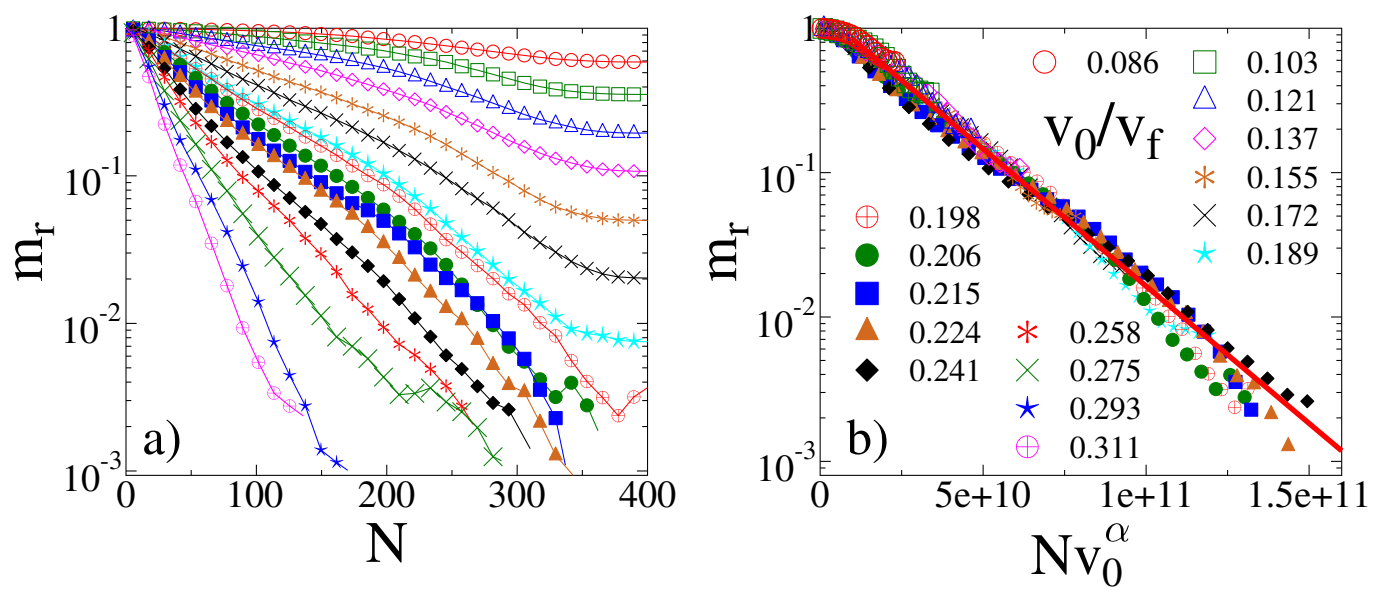

Figure 4. (a) Average mass of the residue $m_{r}$ as a function of the impact number $N$ for several impact velocities $v_{0}$ below the fragmentation critical point $v_{f}$. Panel (b) shows that by rescaling the horizontal axis, curves of different $v_{0}$ values can be collapsed onto one single master curve. Note that data of the lowest impact velocities (highest remaining mass) are in the upper left corner at the start of the master curve. Straight line represents the exponential form of the scaling function $\widetilde{m}_{r}(x)$ of Eq. (1). The legend for (a) and (b) is given in $(b)$.

the threshold velocity above which the asymptotic residual mass is zero even at finite energy threshold for the creation of cracks. In our discrete element model, a complete destruction of the sample is reached when the largest fragment comprises solely a single particle of the discretization. For real materials this state is realized when the residual size approaches a characteristic length scale of the meso-structure, e.g. grain size of materials.

The transition from abrasion to cleavage at the critical velocity $v_{c}$ is driven by the changing mechanism of cracking. In the abrasion phase the dominating mechanism of mass removal is chipping, i.e. crack formation parallel to the contact surface with the wall, which leads to the formation of tiny fragments ${ }^{61,62}$. However, in the case of cleavage, cracks penetrate the solid to significantly deeper regions so that a combination of contact damage and fracture occurs, giving rise to coarser products as well. Additionally, the elastic waves generated by the collision give rise to the gradual accumulation of damage inside the residue which, in turn, can result in fatigue crack growth as the impact sequence proceeds ${ }^{63}$.

Our results demonstrate that above the threshold velocity of micro-cracking $v_{a}$, impact attrition phenomena have additionally two well-defined critical impact velocities $v_{c}$ and $v_{f}$, which separate the three phases of abrasion, cleavage, and fragmentation with distinct qualitative behaviors. The phase diagram of Fig. 2 provides an overview of the distinct qualitative behaviours of impacting solids. For our model solid, the threshold velocities of abrasion and cleavage are $v_{a} / v_{f}=0.124 \pm 0.004$ and $v_{c} / v_{f}=0.224 \pm 0.005$, with respect to the fragmentation critical velocity $v_{f}$.

Sternberg's law and Basquin's law. Figure $4 \mathrm{~b}$ demonstrates that rescaling the impact number $N$ with a proper power $\alpha$ of $v_{0}$, curves belonging to different impact velocities $v_{0}$ can be collapsed on the top of each other, yielding the scaling form

$$
m_{r}\left(N, v_{0}\right)=\widetilde{m}_{r}\left(N v_{0}^{\alpha}\right)
$$

where the scaling function $\widetilde{m}_{r}(x)$ can be approximated by an exponential $\widetilde{m}_{r}(x) \sim \exp (-x)$ (see Fig. $4 \mathrm{~b}$ ), reproducing the time evolution predicted by Sternberg's law ${ }^{48}$. Best collapse is achieved in Fig. $4 \mathrm{~b}$ with the exponent $\alpha=2.1 \pm 0.15$.

It follows from the scaling analysis that increasing the impact velocity $v_{0}$ the characteristic impact number $N_{c}$ of the time evolution decreases as a power law

$$
N_{c} \sim v_{0}^{-\alpha} .
$$

The scaling law Eq. (2) holds in both the abrasion and cleavage phases $v_{a}<v_{0}<v_{f}$ of impact attrition. For cleavage, the characteristic impact number $N_{c}$ can be interpreted as the lifetime of the sample. Since the peak stress, emerging at the contact zone during impact, increases as a power of the impact velocity $v_{0}{ }^{64}$, it follows that the expression (2) of residual lifetime is analogous to the Basquin law of sub-critical fracture phenomena ${ }^{49,50,65-67}$. The Basquin law of fatigue life is a fundamental law of sub-critical fracture. It expresses that under a constant or varying sub-critical load, where the stress amplitude falls below the fracture strength of materials, failure occurs in a finite time which decreases as a power law of the externally applied stress amplitude ${ }^{49}$. Our results demonstrate that the Basquin law holds also for sub-critical impact phenomena.

In the abrasion phase $N_{c}$ characterizes the rate of convergence to the asymptotic residual mass $m_{r}^{a}$. Additionally, the impact velocity also determines the value of $m_{r}^{a}$, which tends to zero when approaching the critical point 


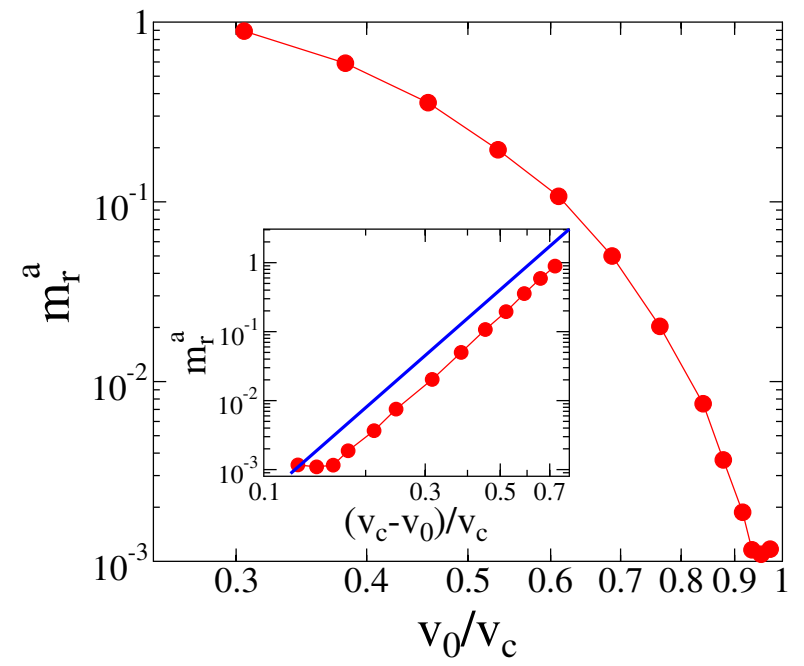

Figure 5. Asymptotic mass $m_{r}^{a}$ of the residue as a function of the impact velocity in the abrasion phase $v_{0}<v_{c}$. Inset: the mass values of the main panel are re-plotted as a function of the relative distance from the critical point $v_{c}$. The straight line represents a power law of exponent $\beta=4.2$.

$v_{c}$ from below, see Fig. 5 which also shows (inset) that the convergence to zero is well described by a power law as a function of the distance from the critical point

$$
m_{r}^{a} \sim\left(v_{c}-v_{0}\right)^{\beta}, \quad \text { for } \quad v_{0} \leq v_{c} .
$$

For the exponent we obtained $\beta=4.2 \pm 0.2$ by fitting of data. Since in the the cleavage phase we have $m_{r}^{a}=0$, whereas in the abrasion phase we have $m_{r}^{a}>0, m_{r}^{a}$ can be considered as the order parameter of the abrasioncleavage phase transition, and $\beta$ is the order parameter exponent of the transition.

Shape evolution: geometric phases inside the abrasion energy phase. Mean field models. In case of polyhedral initial samples, in the abrasion phase we expect that at the beginning of the impact sequence sharp corners and edges are gradually removed, giving rise to an evolution towards an asymptotic rounded shape. In the cleavage phase, due to the breaking of coarser pieces this evolution is more erratic and eventually results in an ultimate destruction. Due to the small size of fragments, we expect that geometric aspects of the abrasion phase may be well reflected in the solutions of averaged, mean field geometric PDE models of attrition $^{43,44}$. The simplest, two-dimensional version of these PDE models may be written as

$$
V=c \kappa,
$$

where $V$ denotes the speed by which a surface points moves inward along the surface normal, $\kappa$ is the scalar curvature and Eq. (4) is often referred to ${ }^{40,41}$ as the curve shortening flow, or as a geometric heat equation, referring to a class of geometrically defined, parabolic partial differential equations which have been, ever since the groundbreaking work of Mullins ${ }^{68,69}$, broadly used in surface evolution models ${ }^{43,70}$. The constant $c$ can be regarded as scaling of time and plays no role if evolution is plotted as a function of the normalized residual mass $m_{r}$. From the mathematical perspective, if we restrict ourselves to classical solutions of Eq. (4) then, instead of considering polyhedra as initial data, we rather consider smooth approximating sequences from which we can pick initial conditions arbitrarily close to a polyhedron. In the approximating sequence all first and second derivatives are continuous.

We remark that Eq. (4) is written in a compact, invariant notation, details about this and other notations are given in Section 1 of the Supplementary Information. The 3D version of this impact-induced attrition model, called the Gauss curvature flow was first introduced by Firey ${ }^{43}$ and its convergence to the sphere was ultimately proven by Andrews ${ }^{71}$. One advantage of the compact notation of Eq. (4) is that the 3D version can be described by an analogous formula, for details see Supplementary Information. Next we will show that these expectations are well founded and PDE models serve indeed as good approximations of impact-induced attrition processes, however, only in the abrasion phase.

Shape descriptors. To give a quantitative characterization of the rounding process, we picked three dimensionless descriptors of the overall shape of the residue which not only provide efficient monitoring of the geometric evolution but also admit meaningful comparison with earlier results: axis ratios, circularity (isoperimetric ratio) and intact surface ratio. 
1. Axis ratios $c / a$ and $b / a$ are traditional geological descriptors ${ }^{9}$ characterizing the shape of the residue ${ }^{25,72}$ where $a>b>c$ refer to the axes of the bounding box of the residue, aligned with the edges of the initial (cuboid) sample.

2. Isoperimetric ratio or circularity of a planar object is given as $R=4 \pi A / P^{2}$, where $A, P$ refer to area and perimeter, respectively. It has been observed ${ }^{8}$ that circularity of the largest projection of sedimentary particles shows universal features in fluvial abrasion and its evolution is entirely determined by the mass loss during impact induced attrition processes. In our DEM, $A$ and $P$ of the residue were obtained as the area and perimeter of the convex hull of the point cloud of the largest projection of the spherical particles of the relaxed body. For more details on shape descriptors see Subsection 1.3 of the Supplementary Information.

3. Intact surface ratio $S / S_{0}$, expressing the intact fraction of the initial surface, was selected following an idea of Richard Hamilton ${ }^{46}$ who, in one of the papers dedicated to the study of curvature-driven flows (leading ultimately towards to his seminal contribution to the proof of the Poincaré - conjecture) describes a curious nonlinear phenomenon about intact surface ratio in the Gauss curvature flow which is the 3D version of (4): he predicted that $S / S_{0}$ will drop to zero after a finite time, marking the end of the first geometric phase for cuboids.Hamilton's result inspired further, detailed research on other curvature-driven PDEs ${ }^{73}$ which found that whether or not flat sides are preserved depends on delicate features of these models. (For more details see Subsection 1.4 of the Supplementary Information.) In the initial state of DEM samples $S_{0}$ is determined as the number of particles covering the external body surface, then the surviving intact surface $S$ is obtained by tracing the particles removed from the initial surface $S_{0}$ in subsequent impacts.

The evolution of axis ratios $c / a$ and $b / a$ and the evolution of the isoperimetric ratio $R$ has been computed in the PDE model ${ }^{9}$ for the very same cuboid initial conditions as in our DEM study. For the evolution of intact surface ratio $S / S_{0}$ in the PDE model we have an analytical result ${ }^{46}$. We will now establish the link between PDE models and microscopic computations by comparing these evolutions. The most striking qualitative feature of the PDE model is the spontaneous emergence of two geometric phases and our computations reveal that these phases are perfectly captured in the microscopic DEM approach. To make the comparison between plots for shape descriptors meaningful, next we seek the corresponding scaling laws.

Scaling laws. Increasing $v_{0}$ accelerates mass removal and thus shape evolution. Figure $6 \mathrm{a}, \mathrm{c}$ demonstrates that both axis ratios $\langle c / a\rangle\left(N, v_{0}\right),\langle b / a\rangle\left(N, v_{0}\right)$ remain initially constant, display sudden growth between the characteristic impact numbers $N_{r}$ and $N_{s}$ and subsequently saturate. Both the overall shape of these functions and their saturation values remain the same in the entire abrasion phase, however both $N_{r}$ and $N_{s}$ decrease with increasing $v_{0}$. We found that rescaling these curves with $v_{0}^{\gamma}$, they collapse onto master curves (see Fig. $6 \mathrm{~b}, \mathrm{~d}$ ) implying the scaling structure

$$
\begin{aligned}
& \langle c / a\rangle\left(N, v_{0}\right)=\Phi\left(N v_{0}^{\gamma}\right), \\
& \langle b / a\rangle\left(N, v_{0}\right)=\Psi\left(N v_{0}^{\gamma}\right),
\end{aligned}
$$

where $\Phi(x)$ and $\Psi(x)$ denote the scaling functions. This also implies that $N_{r}$ and $N_{s}$ both have the same power law dependence

$$
N_{r} \approx A v_{0}^{-\gamma}, \quad N_{s} \approx B v_{0}^{-\gamma},
$$

where the exponent $\gamma$ was obtained numerically $\gamma=3.0 \pm 0.07$. The saturation values $\langle c / a\rangle \approx 0.865$ and $\langle b / a\rangle \approx 0.925$ show that the asymptotic stable shape of the object is slightly anisotropic which may be a consequence of the finite number of the non-breakable discrete elements in the simulation. Our simulations revealed that under the condition of isotropic impacts, the origin of the universal scaling forms is that the shape of the evolving object is controlled by the total relative mass $\mu(N)=1-m_{r}$ lost in $N$ repeated collisions. Recently, it has been suggested ${ }^{8}$ that $\mu(N)$ is also controlling the evolution of the circularity $R$, so henceforth we use this representation for all shape descriptors.

Geometric phases. The PDE model (4) predicts for the evolution of cuboid blocs with moderate initial axis ratios the emergence of two geometric phases: in phase 1 axis ratios $c / a, b / a$ remain approximately constant while roundness increases steeply and saturates close to 1 . In phase 2 the opposite happens: axis ratios increase steeply and saturate close to 1 while roundness remains constant. The conceptual plot of this evolution (as a function of the relative abraded mass $\mu=1-m_{r}$ ) is shown in Fig. 7b1, accompanied by conceptual contours of the specimen, projected along the shortest $(c)$ axis (b2) and representative snapshots of DEM simulations (b3). Figure $7 \mathrm{c} 1$ shows the same plot for $b / a$ and $R$, obtained from the numerical computation ${ }^{9}$ of the PDE (4). Figure 7 (c2) presents the hand-drawn sketch of Hamilton ${ }^{46}$ of his analytical result on the same PDE: intact surface area $S / S_{0}$ survives for a finite time and this marks geometric phase 1 .

In Fig. 7a we compare the DEM computations to the aforementioned analytical predictions. In Fig. 7al we show evolutions of the average axis ratio $\langle b / a\rangle$ and roundness $\langle R\rangle$ in the abrasion energy phase $v_{a}<v_{0}<v_{c}$. Note that curves of different impact velocities all fall on the top of each other in agreement with the scaling collapse predicted in the previous section. It is apparent that we have good qualitative agreement with Fig. 7c1: $\langle b / a\rangle$ remains constant at the initial value $\langle b / a\rangle=1.2 / 1.4$ until $\mu^{*} \approx 0.34$ while $\langle R\rangle$ increases sharply and the opposite can be observed for $\mu>0.34$. Based on this observations we can clearly record the presence of the two geometric phases in the abrasion energy phase for the evolutions of the axis ratios and the roundness. 

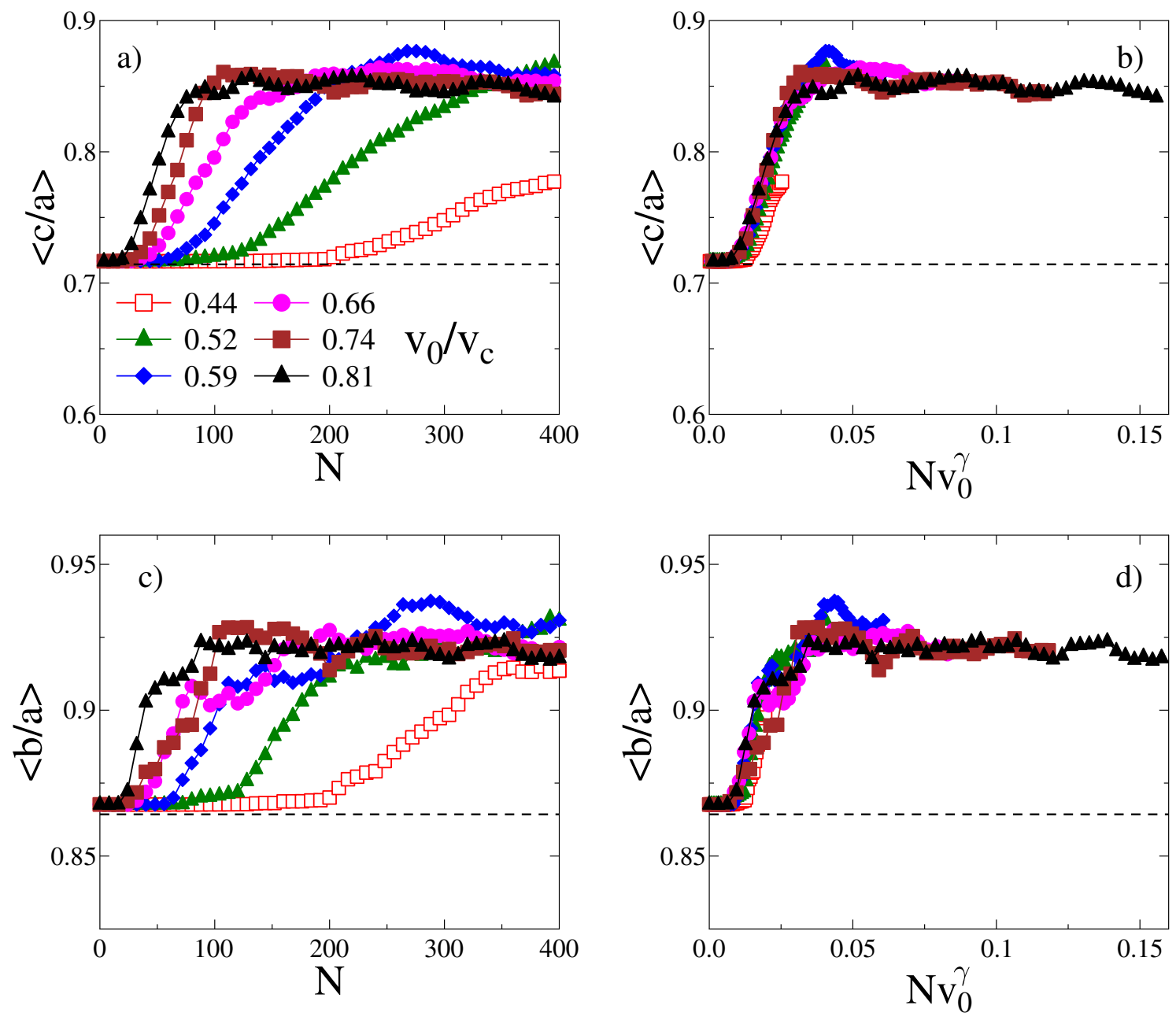

Figure 6. Average side length ratios $\langle c / a\rangle$ (a) and $\langle b / a\rangle$ (c) of the bounding box of the residues as function of the impact number $N$ for different impact velocities inside the abrasion energy phase $v_{0}<v_{c}$. The horizontal dashed lines represent the initial values $\left\langle c_{0} / a_{0}\right\rangle=1 / 1.4$ and $\left\langle b_{0} / a_{0}\right\rangle=1.2 / 1.4$. By rescaling the impact number $N$ in (b) and (d) by an appropriate power $\gamma$ of the impact velocity $v_{0}$, the curves of different $v_{0}$ of (a) and (c) can be collapsed on master curves. Best collapse is achieved using the same exponent $\gamma=3$ in (b) and (d).

In Fig. $7 \mathrm{a} 2$ we show the evolution of the intact surface ratio $S / S_{0}$, also in the abrasion energy phase $v_{a}<v_{0}<v_{c}$. We can observe that this shape descriptor drops to zero at the same relative abraded mass value $\left(\mu^{*} \approx 0.34\right.$ ) which separates the two phases for the evolution of axis ratios and roundness. This is in agreement with the prediction of Hamilton ${ }^{46}$ who claimed that intact surface area will survive for a finite time. It is easy to see that as long as intact surface area exists, the corresponding axis ratio of the cuboid (computed from the bounding box) will remain constant so here again we see a perfect match between the DEM computations and the prediction based on the PDE. The transition point $\mu^{*}$ between the two geometric phases in the microscopic DEM and macroscopic mean field PDE descriptions of shape evolution have a very good agreement.

This confirms our claim that in the abrasion energy phase $v_{a}<v_{0}<v_{c}$ the PDE model offers adequate description of the shape evolution. In sharp contrast, Fig. 7a3 illustrates the evolution of the axis ratio $b / a$ and roundness $R$ in the cleavage energy phase $v_{c}<v_{0}<v_{f}$, both displaying a non-smooth behavior: here we do not expect any mean-field PDE model to provide an adequate description.

\section{Discussion}

Impact induced attrition processes cover a broad variety of phenomena ranging from the gentle removal of fine powder from the surface of rock pieces by low velocity impacts to the immediate disruption of objects in energetic collisions. Understanding gradual mass removal due to a sequence of impact events is crucial in sedimentology since pebbles can be considered as witnesses of the geological conditions of their creation. Universal scaling laws of lifetime, size, and shape of evolving particles are indispensable to decode the information imprinted in pebbles $^{1-4,18}$. In the initial state of this evolution process freshly fragmented rocks are generated ${ }^{25}$ by dynamic breakup of rock masses due to high velocity impacts. While the theory of single impact of solid particles with a hard wall is well understood at the level of particle-based models, impact sequences have been so far only 
a) DEM MODEL

(a1)

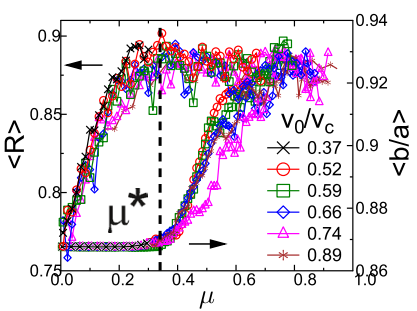

(a2)

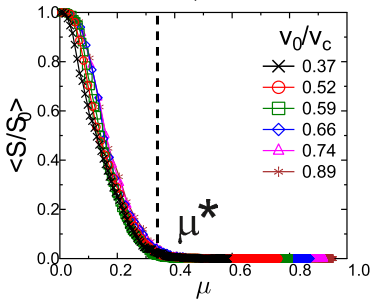

(a3)

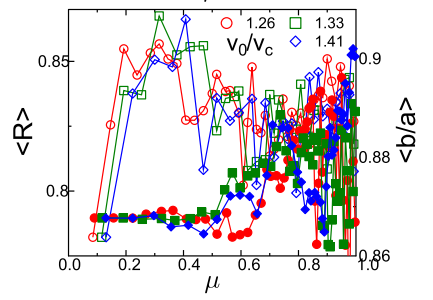

b) CONCEPTUAL DIAGRAM

(b1)

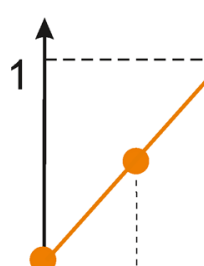

Roundness $<\mathrm{R}>$

(b2)

(b3)

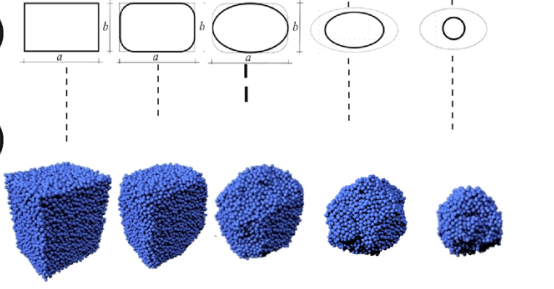

(c1)
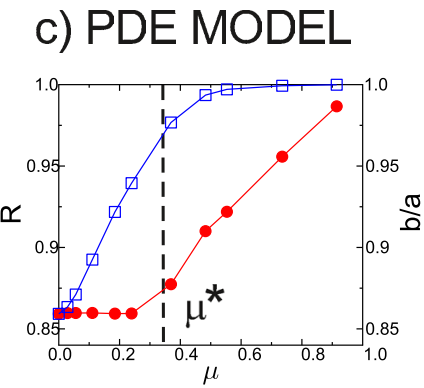

(c2)

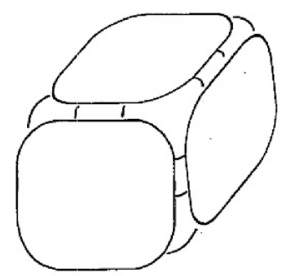

Figure 7. Geometric shape evolution as a function of the relative abraded mass $\mu$. (a) DEM simulations: (a1) Abrasion energy phase: evolution of the average circularity $\langle R\rangle$ and axis ratio $\langle b / a\rangle$. Observe two geometric phases: phase 1 with approximately constant $\langle b / a\rangle$ followed by phase 2 with approximately constant $\langle R\rangle$. Transition at $\mu^{*} \approx 0.34$. (a2) Abrasion energy phase: evolution of intact surface ratio $S / S_{0}$. Transition between phases at $\mu^{*} \approx 0.34$. (a3) Cleavage phase: evolution of the average circularity $\langle R\rangle$ (open symbols) and axis ratio $\langle b / a\rangle$ (filled symbols). Observe absence of smooth evolution. (b1) Schematic, bilinear approximation of two-phase geometric evolution of axis ratios and roundness. (b2) Schematic side view of abrading cuboids, projected along the shortest $(c)$ axis. (b3) Snapshots of DEM simulations. (c) PDE model results: (c1) Evolution of circularity $R$ and axis ratio $b / a^{9}$. Observe two geometric phases: phase 1 with approximately constant $b / a$ followed by phase 2 with approximately constant $R$. (c2) Hand-drawn sketch by R. Hamilton ${ }^{46}$ predicting phase 1 characterized by nonzero intact surface ratio.

modeled by mean field theory which necessarily included gross simplifications of the breaking process. Here we offered the first link between particle-based models and mean field theory for collision sequences.

The main methodological novelty of our study is that we use the discrete element method to realistically simulate the entire physical process of all the individual impacts of long sequences without any additional assumption. Although at high computational costs (by simulating $\approx 5 \times 10^{5}$ collisions with samples consisting of $\approx 12,000$ discrete elements), this approach enabled us to unveil the rich phase structure of impact induced attrition processes. Based on experimental observations, a descriptive classification of single impact breakage has been proposed in ${ }^{19}$, where low, intermediate, and high velocity ranges were distinguished according to the amount and structure of the resulting damage of the body. Here we demonstrated that in multiple impact processes these regimes are separated by universal phase transitions. In addition to the already known damage and fragmentation phases (separated by the critical impact velocity $v_{f}$ ) we identified the abrasion and cleavage phases inside the damage phase (separated by the critical velocity $v_{c}$ ). Abrasion results in finite asymptotic mass (analogous to Sternberg's Law ${ }^{48}$ ) while cleavage results in a complete destruction after a finite number of impacts, with sample lifetime decreasing as a power law of the impact velocity (analogously to Basquin's law).

By identifying the abrasion energy phase we were able to provide the link between microscopic, particlebased models and mean-field curvature-driven equations. We showed that the latter can be regarded as adequate approximations of the former, however, only in the abrasion phase. Our simulations revealed an astonishing universality of the evolution of rounding of the residue. Both the axis ratios and the circularity of the largest projection proved to be entirely determined by the attrition mass: evolutions at different impact velocities $v_{0}$ can be collapsed onto a single curve by rescaling the number of impacts with a proper power $\alpha$ (also called the lifetime exponent) of $v_{0}$. This universality confirmed earlier conjectures and observations ${ }^{8,9}$ on the existence of two geometric phases and also helped to identify a scaling law of the dynamics: the characteristic event number of the onset of shrinking of initially angular objects proved to decrease as a power law of the impact velocity. We were also able to verify a curious effect of geometric nonlinearity, first predicted by Hamilton ${ }^{46}$ : in case of polyhedral initial shapes, a finite amount of the initial surface area survived abrasion for a finite amount of time.

Our findings also fit into the broader picture of efforts to approximate PDE models by microscopic, particlebased simulations. In the context of curvature-driven surface evolution, closest to our current topic, Monte-Carlo 
simulations of the Kardar-Parisi-Zhang (KPZ) equation proved to be a powerful tool to understand the global dynamics ${ }^{74,75}$. However, in contrast to our approach, discrete KPZ models do not use a mechanics-based DEM Kernel and most often they are aimed at surface growth in an orthogonal $[x y z]$ frame.

It is important to emphasize that the excellent qualitative and quantitative agreement (e.g. for the transition point between the two geometric phases) of the microscopic DEM and macroscopic PDE descriptions of shape evolution were obtained without any parameter tuning of DEM simulations. This confirms the high degree of robustness of the results for the broad class of heterogeneous brittle materials. For the initial state of shape evolution we considered mildly anisotropic cuboids, since it has proven to be the generic average shape of freshly fractured rocks ${ }^{45}$. Cuboids with other axis ratios would only change the time scale of shape evolution and shift the transition point $\mu^{*}$ between the geometric phases. Inside the energy phases of abrasion and cleavage, the temporal evolution of mass and shape is controlled by the impact velocity which we could cast into scaling laws. The value of the scaling exponent of lifetime (cleavage) $\alpha$ falls close to 2 , while the exponent $\gamma$ controlling the shape evolution (abrasion) has a higher value $\gamma \approx 3$. Based on fracture mechanics, approximate analytical expressions have been derived for the threshold velocities of the onset of abrasion $v_{a}$ and fragmentation $v_{f}^{62}$. These calculations showed that the critical velocities separating the energy phases of impact attrition phenomena depend on material properties as well as on the mass and linear extension of the sample ${ }^{62}$. Based on the analogy to continuous phase transitions and on the good quantitative agreement between the PDE and DEM approaches for the transition point $\mu^{*}$, we conjecture that the critical exponents $\alpha, \beta$, and $\gamma$ are universal, they depend neither on mechanical, nor on geometrical features of the system.

\section{Methods}

Discrete element model. We performed computer simulations of the repeated sub-critical impact of solid bodies against a hard wall in the framework of a discrete element model of heterogeneous brittle materials which has been successfully applied before to investigate fracture and fragmentation under various types of loading conditions ${ }^{51-54}$. In the model the sample is represented as a random packing, consisting, on the average, of 12,000 spherical particles with a uniformly distributed diameter $d$ in a narrow interval $\Delta d$ around the average $\langle d\rangle$ with $\Delta d /\langle d\rangle=0.05^{53}$. The initial packing is generated by sedimenting the randomly sized particles in a rectangular container which provides a high quality representation of the disordered isotropic micro-structure of rocks. Cohesive interaction is realized by beam elements which connect the particles along the edges of Delaunay triangles constructed from the initial particle positions. In three dimensions (3D) the total deformation of a beam is calculated as the superposition of elongation, torsion, as well as, bending and shearing ${ }^{76}$. Cracks are formed when overstressed beams break according to a physical breaking rule. The breaking condition takes into account the stretching and shearing of particles contacts. The interaction of contacting particles which are not connected by beams is described by the Hertz contact law ${ }^{76}$. In the model the random packing of particles is the only source of disorder which determines the physical properties of beams such as length, cross section, elastic moduli, and moments, as well. At the broken beams along the surface of the spheres cracks are generated inside the solid and as a result of the successive beam breaking fragments are formed. The time evolution of the fragmenting solid is obtained by solving the equations of motion of the individual particles with proper initial and boundary conditions. The model has been validated by comparing $(i)$ the stress field generated in body wall collisions to finite element calculations $s^{30,56}$, and (ii) the crack structure and fragment mass distributions to the experimental findings ${ }^{57-59}$. Further details of the model construction and of the parameter setting can be found in the Supplementary Information and in ${ }^{53,55}$

Simulations of body-wall collisions. We used the model to simulate the impact of a rectangular body with a hard wall. The initial state of the simulations was prepared by placing the cubic sample close to a hard wall with a random orientation assigning the same initial velocity to the particles pointing perpendicular to the wall. As the body moves, its particles overlap with the wall and experience an elastic restoring force according to the Hertz contact law ${ }^{64,76}$ giving rise to deformation and cracking of the body.

Preparation of the residue for repeated impacts. In the final state of an impact process particles of the fragments are not completely relaxed in the sense that the fragments can be deformed and would gradually relax by dissipating energy due to the internal friction of the material captured as a viscous damping force between contacting particles. In order to reduce the computational time, we identify the particles of the residue inside the original sample and replace it by its relaxed counterpart. For each collision during the sequence, the residue is randomly rotated in the initial state to avoid any directional dependence. At each impact number, averages over 120 samples, i.e. over 120 impact directions, were performed, which ensured the high quality of the results even for small residual sizes where the surviving particle clusters have an irregular shape.

Figures 2, 4, 5 and 6 were generated using Graphics Layout Engine GLE 4.2.5 (http://glx.sourceforge.net/), Figs. 1 and 3 were created with POV-Ray 3.7.0 (http://www.povray.org/), and Fig. 7 was made using CorelDRAW Graphics Suit 2019 (https://www.coreldraw.com/). All figures were made by the authors.

\section{Data availability}

Mass data and shape descriptors of residues obtained from DEM simulations and PDE computations are freely available in the OSF Data repository at https://osf.io/g2ftd/.

\section{Code availability}

The numerical code used for data evaluation in this paper is available from the corresponding author upon reasonable request. 
Received: 5 June 2021; Accepted: 5 October 2021

Published online: 19 October 2021

\section{References}

1. Wald, Q. R. The form of pebbles. Nature 345, 211 (1990).

2. Lorang, M. S. \& Komar, P. D. Pebble shape. Nature 347, 433-434 (1990).

3. Turcotte, D. L. Fractals and Chaos in Geology and Geophysics (Cambridge University Press, Cambridge, 1997).

4. Durian, D. J. et al. What is in a pebble shape?. Phys. Rev. Lett. 97, 028001 (2006).

5. Williams, R. M. E. et al. Martian fluvial conglomerates at gale crater. Science 340, 1068-1072 (2013).

6. Doan, M.-L. \& Gary, G. Rock pulverization at high strain rate near the San Andreas fault. Nat. Geosci. 2, 709-712 (2009)

7. Dufek, J., Manga, M. \& Patel, A. Granular disruption during explosive volcanic eruptions. Nat. Geosci. 5, 561-564 (2012).

8. Novák-Szabó, T. et al. Universal characteristics of particle shape evolution by bed-load chipping. Sci. Adv. 4, eaao4946 (2018).

9. Domokos, G., Jerolmack, D., Sipos, A. \& Török, A. How river rocks round: Resolving the shape-size paradox. PLoS ONE 9, e88657 (2014).

10. Jones, T. J. \& Russell, J. K. Ash production by attrition in volcanic conduits and plumes. Sci. Rep. 7, 1-12 (2017).

11. Hornby, A. J. et al. Phase partitioning during fragmentation revealed by QEMSCAN particle mineralogical analysis of volcanic ash. Sci. Rep. 9, 1-12 (2019).

12. Kok, J. F. A scaling theory for the size distribution of emitted dust aerosols suggests climate models underestimate the size of the global dust cycle. Proc. Natl. Acad. Sci. 108, 1016-1021 (2011).

13. Dahmen, K. A., Ben-Zion, Y. \& Uhl, J. T. A simple analytic theory for the statistics of avalanches in sheared granular materials. Nat. Phys. 7, 554-557 (2011).

14. Farinella, P. \& Zappalà, V. The shapes of the asteroids. Adv. Space Res. 19, 181-186 (1997).

15. Durda, D. D. et al. The shapes of fragments from catastrophic disruption events: Effects of target shape and impact speed. Planet. Space Sci. 107, 77-83 (2015).

16. Domokos, G., Sipos, A. Á., Szabó, G. M. \& Várkonyi, P. L. Formation of sharp edges and planar areas of asteroids by polyhedral abrasion. Astrophys. J. 699, L13-L16 (2009).

17. Domokos, G., Sipos, A. Á., Szabó, G. M. \& Várkonyi, P. L. Explaining the elongated shape of 'oumuamua by the eikonal abrasion model. Res. Notes AAS 1, 50 (2017).

18. Szabó, T., Domokos, G., Grotzinger, J. P. \& Jerolmack, D. J. Reconstructing the transport history of pebbles on Mars. Nat. Commun. 6, 8366 (2015)

19. Salman, A. et al. Descriptive classification of the impact failure modes of spherical particles. Powder Technol. 143-144, 19-30 (2004)

20. Wills, B. A. \& Finch, J. A. Chapter 5-Comminution. In Wills, B. A. \& Finch, J. A. (eds.) Wills' Mineral Processing Technology, 8th edn, 109-122 (Butterworth-Heinemann, Boston, 2016).

21. Spahn, F., Neto, E. V., Guimarães, A. H. F., Gorban, A. N. \& Brilliantov, N. V. A statistical model of aggregate fragmentation. New J. Phys. 16, 013031 (2014).

22. Brilliantov, N. et al. Size distribution of particles in Saturn's rings from aggregation and fragmentation. Proc. Natl. Acad. Sci. 112, 9536-9541 (2015).

23. Andrews, E. \& Kim, K.-S. Threshold conditions for dynamic fragmentation of glass particles. Mech. Mater. 31, 689-703 (1999).

24. Hauk, T., Bonaccurso, E., Roisman, I. V. \& Tropea, C. Ice crystal impact onto a dry solid wall. particle fragmentation. Proc. Roy. Soc. A Math. Phys. Eng. Sci. 471, 20150399 (2015).

25. Domokos, G., Kun, F., Sipos, A. A. \& Szabó, T. Universality of fragment shapes. Sci. Rep. 5, 9147 (2015).

26. Kun, F. \& Herrmann, H. J. Transition from damage to fragmentation in collision of solids. Phys. Rev. E 59, 2623 (1999).

27. Katsuragi, H., Sugino, D. \& Honjo, H. Scaling of impact fragmentation near the critical point. Phys. Rev. E 68, 046105 (2003).

28. Kadono, T., Arakawa, M. \& Mitani, N. Fragment velocity distribution in the impact disruption of thin glass plates. Phys. Rev. E 72, 045106(R) (2005).

29. Aström, J. A. Statistical models of brittle fragmentation. Adv. Phys. 55, 247-278 (2006).

30. Carmona, H. A., Wittel, F. K., Kun, F. \& Herrmann, H. J. Fragmentation processes in impact of spheres. Phys. Rev. E 77, 051302 (2008).

31. Wang, Y. \& Tonon, F. Dynamic validation of a discrete element code in modeling rock fragmentation. Int. J. Rock Mech. Min. Sci. 48, 535-545 (2011).

32. Timár, G., Kun, F., Carmona, H. A. \& Herrmann, H. J. Scaling laws for impact fragmentation of spherical solids. Phys. Rev. E 86, 016113 (2012).

33. Ye, Y., Thoeni, K., Zeng, Y., Buzzi, O. \& Giacomini, A. Numerical investigation of the fragmentation process in marble spheres upon dynamic impact. Rock Mech. Rock Eng. (2019).

34. Ma, G., Zhou, W., Zhang, Y., Wang, Q. \& Chang, X. Fractal behavior and shape characteristics of fragments produced by the impact of quasi-brittle spheres. Powder Technol. 325, 498-509 (2018).

35. Arbiter, N., Harris, C. \& Stamboltzis, A. G. Single fracture of brittle spheres. Trans. Soc. Min. Eng. AIME 244, 118-244 (1969).

36. Chau, K. T., Wei, X. X., Wong, R. H. C. \& Yu, T. X. Fragmentation of brittle spheres under static and dynamic compressions: Experiments and analyses. Mech. Mater. 32, 543-554 (2000).

37. Myagkov, N. On critical exponents for impact fragmentation of spherical solids. Phys. A Stat. Mech. Its Appl. 534, 122117 (2019).

38. Timár, G., Blömer, J., Kun, F. \& Herrmann, H. J. New universality class for the fragmentation of plastic materials. Phys. Rev. Lett. 104, 095502 (2010).

39. Moukarzel, C. F., Fernandez-Sabido, S. F. \& Ruiz-Suarez, J. C. Phase transition in liquid drop fragmentation. Phys. Rev. E 75, 061127 (2007).

40. Gage, M. \& Hamilton, R. The heat equation shrinking convex plane curves. J. Differ. Geom. 23, 69-96 (1986).

41. Grayson, M. The heat equation shrinks embedded plane curves to round points. J. Differ. Geom. 26, 285-314 (1987).

42. Perelman, G. Ricci flow with surgery on three-manifolds. ArXiv preprint https://arxiv.org/abs/math/0303109 (2003).

43. Firey, W. Shapes of worn stones. Mathematika 21, 1-11 (1974).

44. Bloore, F. J. The shape of pebbles. Math. Geol. 9, 113-122 (1977).

45. Domokos, G., Jerolmack, D. J., Kun, F. \& Török, J. Plato's cube and the natural geometry of fragmentation. Proc. Natl. Acad. Sci. 117, 18178-18185 (2020).

46. Hamilton, R. Worn stones with flat sides. Discourses Math. Appl. 3, 69-78 (1994).

47. Priour Jr., D. Time scales for rounding of rocks through stochastic chipping. ArXiv preprint https://arxiv.org/abs/2003.03476 (2020).

48. Sternberg, H. Untersuchungen über längen- und querprofil geschiebeführender Flüsse. Zeitschr. Bauwesen 25, 483-506 (1875).

49. Basquin, O. H. The exponential law of endurance tests. Proc. Am. Soc. Test. Mater. ASTEA 10, 625 (1910).

50. Kun, F., Carmona, H. A., Andrade, J. S. Jr. \& Herrmann, H. J. Universality behind basquin's law of fatigue. Phys. Rev. Lett. 100, 094301 (2008) 
51. Kun, F., Varga, I., Lennartz-Sassinek, S. \& Main, I. G. Approach to failure in porous granular materials under compression. Phys. Rev. E 88, 062207 (2013).

52. Kun, F., Varga, I., Lennartz-Sassinek, S. \& Main, I. G. Rupture cascades in a discrete element model of a porous sedimentary rock. Phys. Rev. Lett. 112, 065501 (2014).

53. Pál, G., Varga, I. \& Kun, F. Emergence of energy dependence in the fragmentation of heterogeneous materials. Phys. Rev. E 90, 062811 (2014).

54. Pál, G., Jánosi, Z., Kun, F. \& Main, I. G. Fragmentation and shear band formation by slow compression of brittle porous media. Phys. Rev. E 94, 053003 (2016).

55. Pál, G. \& Kun, F. Mass-velocity correlation in impact induced fragmentation of heterogeneous solids. Granul. Matter 18, 74 (2016).

56. Wittel, F., Carmona, H., Kun, F. \& Herrmann, H. Mechanisms in impact fragmentation. Int. J. Fract. 154, 105 (2008).

57. Majzoub, R. \& Chaudhri, M. High-speed photography of low-velocity impact cracking of solid spheres. Phil. Mag. Lett. 80, 387-393 (2000).

58. Salman, A. et al. An experimental investigation of particle fragmentation using single particle impact studies. Powder Technol. 128, 36-46 (2002).

59. Schönert, K. Breakage of spheres and circular discs. Powder Technol. 143-144, 2-18 (2004).

60. Kadono, T. Fragment mass distribution of plate like objects. Phys. Rev. Lett. 78, 1444-1447 (1997).

61. Subero, J. \& Ghadiri, M. Breakage patterns of agglomerates. Powder Technol. 120, 232-243 (2001).

62. Ghadiri, M. \& Zhang, Z. Impact attrition of particulate solids. Part 1: A theoretical model of chipping. Chem. Eng. Sci. 57, 3659-3669 (2002).

63. Herrmann, H. J. \& Roux, S. (eds) Statistical Models for the Fracture of Disordered Media. Random Materials and Processes (Elsevier, Amsterdam, 1990).

64. Johnson, K. L. Contact Mechanics (Cambridge University Press, New York, 1985).

65. Suresh, S. Fatigue of Materials (Cambridge University Press, Cambridge, 1998).

66. Sornette, D., Magnin, T. \& Brechet, Y. The physical origin of the coffin manson law in low-cycle fatigue. Europhys. Lett. 20, 433 (1992).

67. Vieira, A. P., Andrade, J. S. \& Herrmann, H. J. Subcritical crack growth: The microscopic origin of Paris' law. Phys. Rev. Lett. 100, $195503(2008)$.

68. Mullins, W. Theory of thermal grooving. J. Appl. Phys. 28, 333-339 (1957).

69. Mullins, W. \& Shewmon, P. The kinetics of grain boundary grooving in copper. Acta Metall. 7, 163-170 (1959).

70. Huisken, G. Asymptotic behavior for singularities of the mean curvature flow. J. Diff. Geom. 31, 285-299 (1990).

71. Andrews, B. Gauss curvature flow: The fate of rolling stones. Invent. Math. 138, 151-161 (1999).

72. Pen, L. M. L., Powrie, W., Zervos, A., Ahmed, S. \& Aingaran, S. Dependence of shape on particle size for a crushed rock railway ballast. Granul. Matter 15, 849-861 (2013).

73. Andrews, B., McCoy, J. \& Zheng, Y. Contracting convex hypersurfaces by curvature. Calc. Var. Partial Differ. Equ. 47, 611-665 (2013).

74. Ódor, G. Universality in Nonequilibrium Lattice Systems: Theoretical Foundations (World Scientific, Singapore, 2008).

75. Ódor, G., Liedke, B. \& Heinig, K.-H. Directed $d$-mer diffusion describing the Kardar-Parisi-Zhang-type surface growth. Phys. Rev. E 81, 031112 (2010).

76. Radjai, F. \& Dubois, F. Discrete-Element Modeling of Granular Materials (Wiley-Iste, London, 2011).

\section{Acknowledgements}

The authors thank András Sipos for his invaluable help with computing Fig. 7(c1). The work is supported by the EFOP-3.6.1-16-2016-00022 project. The project is co-financed by the European Union and the European Social Fund. This research was supported by the National Research, Development and Innovation Fund of Hungary, financed under the K-16 funding scheme Projects No. K 119967 and K 119245 and under the Thematic Excellence Programs of the Ministry for Innovation and Technology in Hungary TKP2021-BME-NVA at the Budapest University of Technology and Economics and TKP2020-IKA-04 at the University of Debrecen.

\section{Author contributions}

G.P. carried out computer simulations. G.P. and F.K. performed the data analysis. F.K. and G.D. conceived and designed the study and drafted the manuscript. All authors read and approved the manuscript.

\section{Competing interests}

The authors declare no competing interests.

\section{Additional information}

Supplementary Information The online version contains supplementary material available at https://doi.org/ 10.1038/s41598-021-00030-1.

Correspondence and requests for materials should be addressed to F.K.

Reprints and permissions information is available at www.nature.com/reprints.

Publisher's note Springer Nature remains neutral with regard to jurisdictional claims in published maps and institutional affiliations.

Open Access This article is licensed under a Creative Commons Attribution 4.0 International License, which permits use, sharing, adaptation, distribution and reproduction in any medium or format, as long as you give appropriate credit to the original author(s) and the source, provide a link to the Creative Commons licence, and indicate if changes were made. The images or other third party material in this article are included in the article's Creative Commons licence, unless indicated otherwise in a credit line to the material. If material is not included in the article's Creative Commons licence and your intended use is not permitted by statutory regulation or exceeds the permitted use, you will need to obtain permission directly from the copyright holder. To view a copy of this licence, visit http://creativecommons.org/licenses/by/4.0/.

(C) The Author(s) 2021 